\title{
Ocorrência de Trypanoxyuris (Trypanoxyuris) minutus (Schneider, 1866) (Nematoda, Oxyuridae) em Alouatta guariba clamitans Cabrera, 1940 (Primates, Atelidae) em Minas Gerais, Brasil
}

Occurrence of Trypanoxyuris (Trypanoxyuris) minutus (Schneider, 1866) (Nematoda, Oxyuridae) in Alouatta guariba clamitans Cabrera, 1940 (Primates, Atelidae) in Minas Gerais, Brazil

Danielle de Paiva Souza ${ }^{1}$; Cecilia Maria da Fonseca Ribeiro Magalhães ${ }^{1}$; Fabiano Matos Vieira ${ }^{2 *}$; Sueli de Souzalima ${ }^{2}$

${ }^{1}$ Universidade Federal de Juiz de Fora - UFJF

${ }^{2}$ Laboratório de Taxonomia e Ecologia de Helmintos, Departamento de Zoologia, Instituto de Ciências Biológicas,

Universidade Federal de Juiz de Fora - UFJF

Recebido em 15 de Setembro de 2009

Aceito em 1 de Outubro de 2009

\section{Resumo}

Este trabalho teve como objetivo registrar a ocorrência de Trypanoxyuris (Trypanoxyuris) minutus em Alouatta guariba clamitans no estado de Minas Gerais, Brasil, e fornecer dados quantitativos sobre as infrapopulaçóes desses nematoides, além de dados morfológicos e biométricos de machos e fêmeas. Dois espécimes de A. guariba clamitans, mortos acidentalmente, foram necropsiados, visando aos estudos parasitológicos. Apenas o intestino grosso e o ceco estavam parasitados por T. (T.) minutus. A intensidade do parasitismo no intestino grosso foi de 6650 parasitos e a densidade de 2,6 parasitos $/ \mathrm{cm}^{3}$ de intestino. No ceco, observou-se uma intensidade média de $6.753 \pm 490,73$ parasitos, com uma densidade média de $6,23 \pm 5,13$ parasitos $/ \mathrm{cm}^{3}$. O presente trabalho fornece informaçóes sobre a biometria dos adultos de $T$. (T.) minutus nessa espécie de hospedeiro, e se constitui no primeiro registro dessa espécie em $A$. guariba clamitans em Minas Gerais, Brasil.

Palavras-chave: Trypanoxyuris (Trypanoxyuris) minutus, Oxyurida, Alouatta guariba clamitans, primatas.

\begin{abstract}
This study aims to register the occurrence of Trypanoxyuris (Trypanoxyuris) minutus in Alouatta guariba clamitans, in Minas Gerais state, Brazil. Two specimens of A. guariba clamitans, died accidentally, have been necropsied for parasitological studies. Only the large intestine and caecum were infected by $T$. minutus. The parasitism intensity was 6650 parasites and the density was 2.6 parasites $/ \mathrm{cm}^{3}$ of large intestine. In the caecum, the mean intensity was $6753 \pm 490.73$ parasites, with mean density of $6.23 \pm 5.13$ parasites $/ \mathrm{cm}^{3}$. The present study supplies information on adult nematodes biometry and this is the first record of T. (T.) minutus in A. guariba clamitans from Minas Gerais State, Brazil.
\end{abstract}

Keywords: Trypanoxyuris (Trypanoxyuris) minutus, Oxyurida, Alouatta guariba clamitans, primates.

Os primatas do gênero Alouatta são parasitados por diversas espécies de helmintos (STUART et al., 1998), dentre as quais destacam-se os nematoides da espécie Trypanoxyuris (Trypanoxyuris) minutus (Schneider, 1866) (Oxyuridae) (STUART et al., 1998), que ocorrem exclusivamente no ceco e intestino grosso de primatas desse gênero (HUGOT; GARDNER; MORAND, 1996; STUART et al., 1998). Os objetivos do presente trabalho foram registrar a ocorrência de $T$. (T.) minutus em A. guariba clamitans no estado de Minas Gerais, Brasil, e fornecer dados quantitativos sobre as

\footnotetext{
*Autor para correspondência: Fabiano Matos Vieira Laboratório de Taxonomia e Ecologia de Helmintos, Departamento de Zoologia, Instituto de Ciências Biológicas, Universidade Federal de Juiz de Fora - UFJF, Campus Universitário, Bairro Martelos, CEP 36.036-330 Juiz de Fora - MG, Brasil; e-mail: fmatosvieira@gmail.com
}

infrapopulaçôes desses nematoides, além de dados morfológicos e biométricos de machos e fêmeas.

Dois espécimes de A. guariba clamitans, do sexo feminino, cedidos pelo Escritório Regional do Instituto Brasileiro do Meio Ambiente e dos Recursos Naturais Renováveis (IBAMA), após morte acidental na BR040, em Juiz de Fora (21 40' 22,03" S e $43^{\circ} 26^{\prime} 39,07^{\prime \prime}$ O), Minas Gerais, Brasil, sob congelamento, foram necropsiados em abril de 2004. Os hospedeiros foram identificados segundo Gregorin (2006). Os nematoides foram fixados em AFA, acondicionados em etanol $70^{\circ} \mathrm{GL}$ e clarificados em Lactofenol para identificação da espécie, que foi feita segundo Yamaguti (1961), Hugot; Vaucher (1985); Hugot; Gardner; Morand (1996) e Vicente et al. (1997). As fotomicrografias foram feitas com câmera digital Sony Cyber-shot ${ }^{\circledast}$ DSC-P52, com 3.2 mega pixels de resolução acoplada ao microscópio Olympus ${ }^{\circledR}$ BX41. As medidas são fornecidas em amplitudes, seguidas das médias 
e desvio-padrão entre parênteses. Os termos ecológicos e os parâmetros quantitativos das infrapopulaçóes foram utilizados de acordo com Bush et al. (1997). Espécimes representativos dos nematoides foram depositados na Coleção Helmintológica do Instituto Oswaldo Cruz (CHIOC), Rio de Janeiro, Brasil.

\section{Trypanoxyuris (Trypanoxyuris) minutus (Schneider, 1866)}

Descrição (17 machos e 17 fêmeas): Nematoides com acentuado dimorfismo sexual, sendo as fêmeas maiores que os machos. Tanto os machos quanto as fêmeas apresentaram a extremidade anterior com placas cefálicas com formato quadrangular (Figuras 1c e 1d), com as aberturas dos anfídios entre as papilas dorsais e subventrais. Em ambos os sexos, há a presença de dois lábios: um dorsal, que é bilobado (Figuras 1c e 1d), e outro ventral simples.

Machos (Figura b). Comprimento total entre 2,15 e 2,69 mm $(2,46 \pm 0,17)$, a largura na região da junção esôfago intestinal entre 105 e $125 \mu \mathrm{m}(111,94 \pm 5,05)$, o comprimento do esôfago (Figura b) entre 556,25 e $775 \mu \mathrm{m}(681,1 \pm 49,97)$, a distância do anel nervoso à extremidade anterior entre 132,83 e 185,07 $\mu \mathrm{m}$
$(161,42 \pm 49,5)$. Possuem um par de asas cefálicas laterais compostas por uma crista. A cauda contêm dois pares de papilas pré-cloacais e 3 pares de papilas pós-cloacais (Figura e), apresenta comprimento entre 11,25 e 17,50 $\mu \mathrm{m}(13,64 \pm 1,89)$, o espículo único (Figura e) com comprimento entre 43,75 e 56,25 $\mu \mathrm{m}(52,05 \pm 2,79)$ e o espinho ou apêndice caudal (Figura e) com comprimento entre 15 e $22,50 \mu \mathrm{m}(18,93 \pm 2,44)$.

Fêmeas (Figura a): Comprimento total entre 5,52 e 7,86 mm $(6,65 \pm 0,69)$, a largura na regiāo da junção esôfago intestinal entre 260 e $385 \mu \mathrm{m}(307,08 \pm 44,79)$, o comprimento do esôfago entre 1,45 e 1,67 mm (1,6 \pm 0,05), a distância do anel nervoso

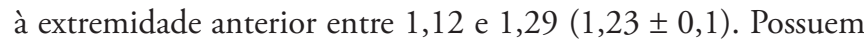
um par de asas cefálicas laterais, entretanto cada asa é composta por uma crista dupla. Distância da vulva à extremidade anterior entre 2,15 e $2,95 \mathrm{~mm}(2,60 \pm 0,23)$ e a cauda (Figura f) com

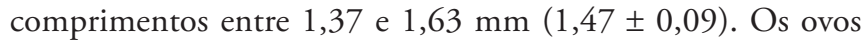
no útero $(\mathrm{n}=170)$ (Figura $\mathrm{g}$ ) possuem comprimentos entre 41,45 e $50,12 \mu \mathrm{m}(47,42 \pm 2,8)$ e larguras entre 21,87 e $24,5 \mu \mathrm{m}$ $(23,45 \pm 0,85)$.
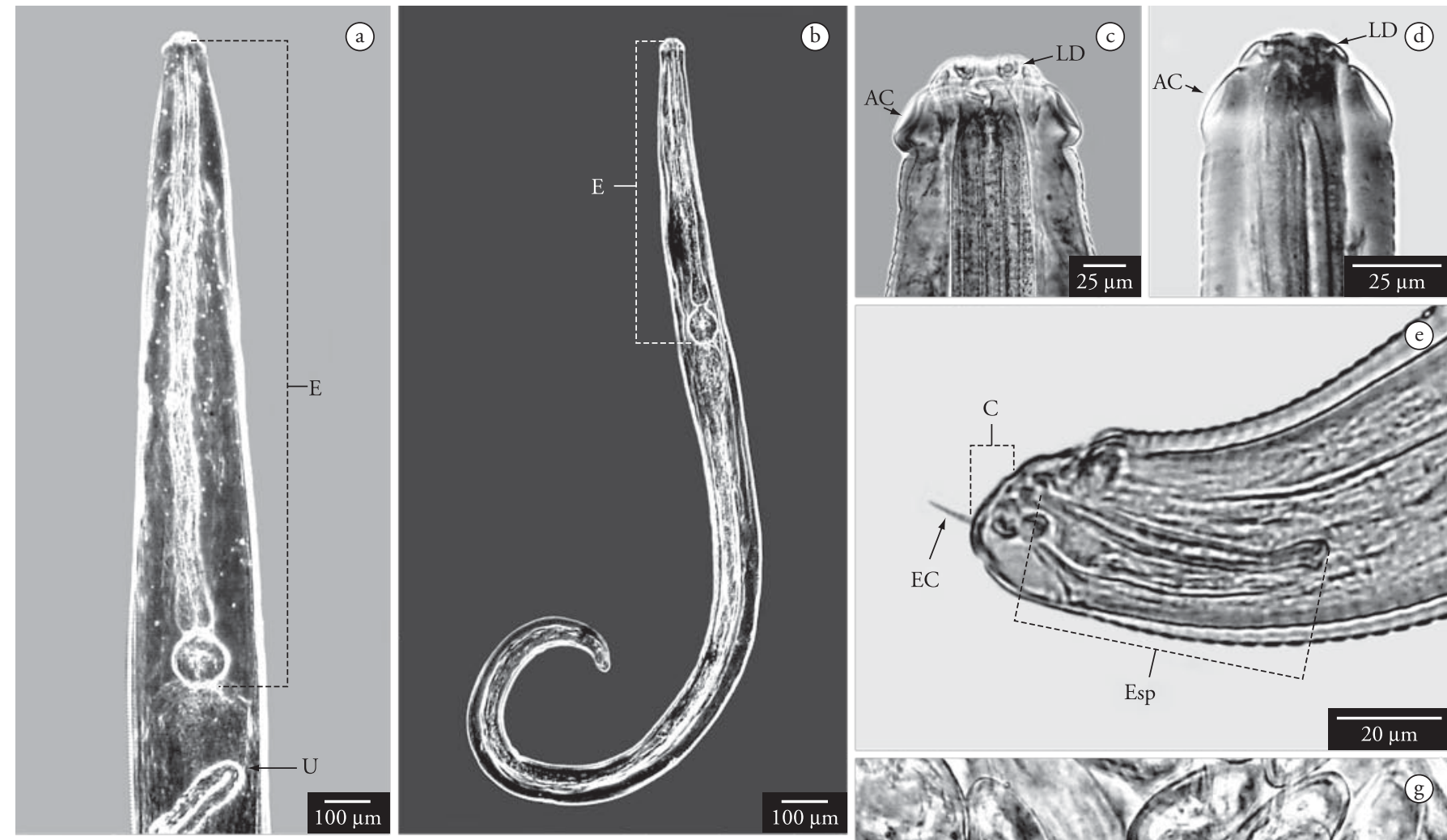

(f)
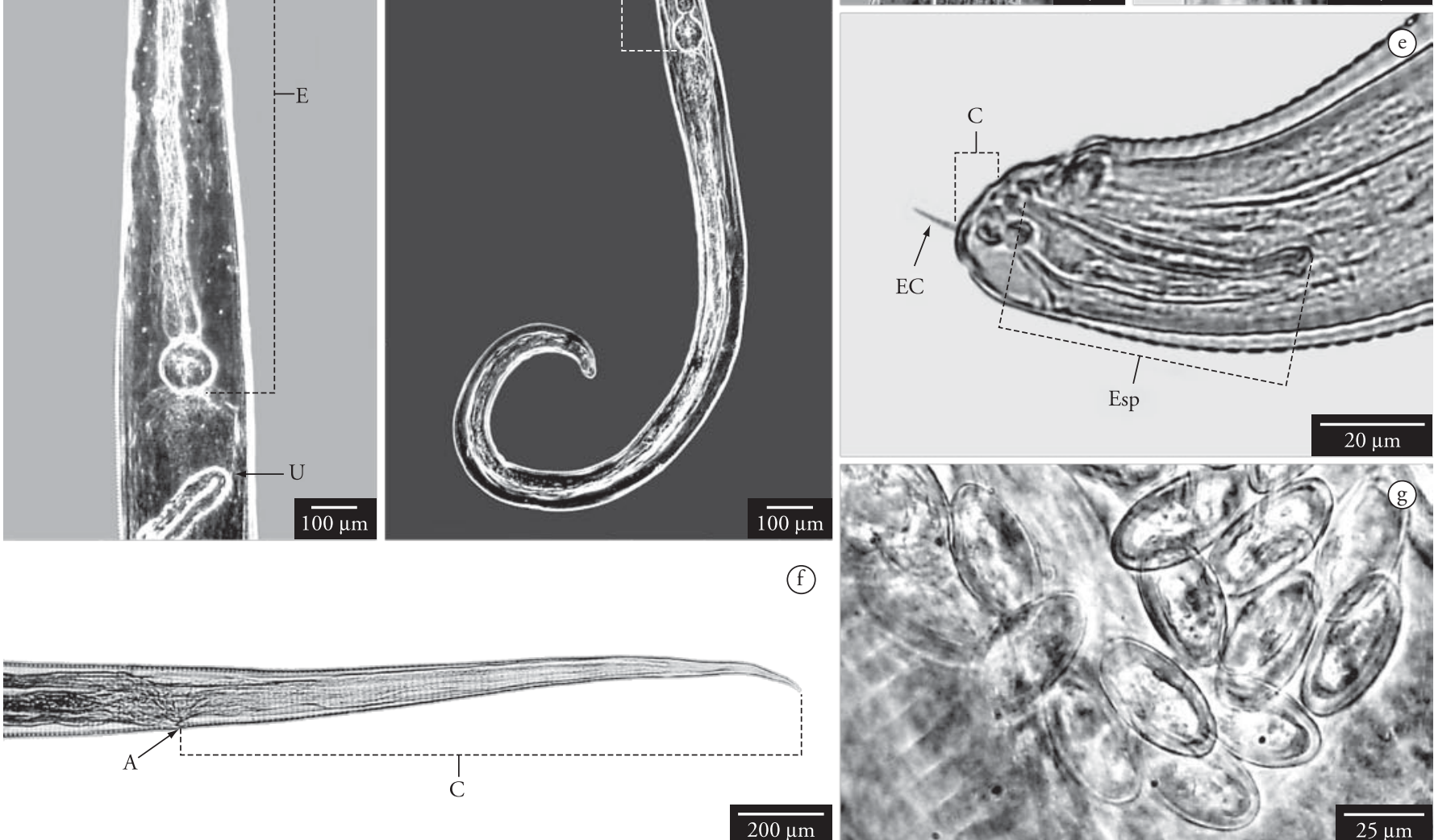

\section{(1)}

Figura 1. Trypanoxyuris minutus. a) Região anterior da fêmea; b) Visão total do macho; c) Extremidade anterior da fêmea; d) Extremidade anterior do macho; e) Região posterior - cauda do macho; f) Região posterior - cauda da fêmea; g) Ovos no útero; (A - ânus; AC - asa cefálica; C - cauda; E - esôfago; LD - lábio dorsal; EC - espinho caudal; Esp. - espículo; U - útero). 
Hospedeiro: Alouatta guariba clamitans Cabrera, 1940 (Primates, Atelidae) (Bugio).

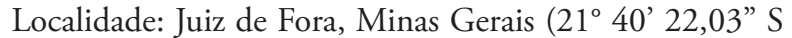
e $43^{\circ} 26^{\prime} 39,07^{\prime \prime} \mathrm{O}$ )

Sítios de infecção: Ceco e intestino grosso

Intensidade parasitária: $6754 \pm 490,73$ parasitos no ceco (dois hospedeiros); 6.650 parasitos no intestino grosso (um hospedeiro).

Densidade parasitária: $6,23 \pm 5,13$ parasitos $/ \mathrm{cm}^{3}$ do ceco; 2,6 parasitos $/ \mathrm{cm}^{3}$ do intestino grosso.

Proporção sexual (machos: fêmeas): 4,90:1 no intestino delgado; 220:1 no intestino grosso.

Espécimes representativos: depositados na CHIOC sob os números 35.647 - 35.649.

Os nematoides do gênero Trypanoxyuris são parasitos exclusivos de primatas das famílias Cebidae e Atelidae (HUGOT; VAUCHER, 1985; HUGOT; GARDNER; MORAND, 1996; STUART et al., 1998). Contudo, o parasitismo por T. minutus é relatado exclusivamente em primatas do gênero Alouatta da América do Sul (STUART et al., 1998; AMATO et al., 2002).

No Brasil, existem os relatos do parasitismo por T. minutus em A. caraya (Humboldt, 1812) (TRAVASSOS; PINTO; MUNIZ, 1927), A. seniculus (Linnaeus, 1766) (TRAVASSOS, 1925), A. belzebul (Linnaeus, 1766) (TRAVASSOS, 1925) e $A$. fusca (Geoffroy, 1812) (= A. guariba guariba) (TRAVASSOS, 1925), e um relato de morte de um espécime de $A$. guariba clamitans, associada à alta intensidade populacional de $T$. minutus no ceco (AMATO et al., 2002). São escassas as informaçóes a respeito da ecologia de infrapopulaçōes de T. minutus em espécies de Alouatta. Amato et al. (2002) registraram a intensidade de 61.870 parasitos no ceco de um espécime de $A$. guariba clamitans (Cabrera, 1940), que é elevada em comparação com o observado no presente trabalho $(6754 \pm 490,73$ parasitos no ceco, $\mathrm{n}=2)$.

No presente estudo, o único hospedeiro que estava parasitado, tanto no ceco como no intestino grosso, apresentou um maior número de fêmeas no ceco $(\mathrm{n}=1350)$ do que no intestino grosso $(\mathrm{n}=30)$. Essa discrepância populacional não foi observada entre os machos nas distintas infrapopulaçóes (ceco $=5.750$; intestino grosso $=6.620)$. Tal fato talvez se explique pelo fato do ceco ser uma região do trato gastrintestinal mais próxima ao ânus, provavelmente um sítio preferencial das fêmeas de oxiurídeos; em razão dessas apresentarem um comportamento migratório em direção ao ânus, para depositarem seus ovos na região perianal (ADAMSON, 1989; ANDERSON, 2000).

Os primatas são particularmente vulneráveis aos efeitos do parasitismo por frequentemente viverem em grupos sociais estreitos, o que facilita a transmissão dos parasitos (STONER, 1996) que, no caso de Trypanoxyuris spp. ocorre pelo hábito da ingestão fecal e consequente ingestão de ovos (STUART et al., 1998). O contágio intraespecífico é favorecido pelo costume dos primatas manterem essa estreita associação entre os indivíduos que formam um grupo (STONER, 1996), o que talvez explique as altas prevalências e intensidades parasitárias em algumas populaçóes de primatas. Não somente a densidade dos primatas pode influenciar as infecçóes parasitárias: o confinamento dos indivíduos em corredores estreitos, resultantes do desmatamento, acaba propiciando uma maior transmissão dos parasitos. Essa constante exposição pode resultar em uma alta prevalência e intensidade dos parasitos, podendo existir, em um longo prazo, consequências na longevidade e na estrutura populacional desses primatas silvestres (STONER, 1996).

No Estado de Minas Gerais, esse nematoide até então não havia sido registrado. Portanto, este trabalho é o primeiro registro de T. (T.) minutus em Minas Gerais.

\section{Agradecimentos}

Ao Escritório Regional do IBAMA em Juiz de Fora, pelo envio dos espécimes de hospedeiros utilizados no presente trabalho.

\section{Referências}

ADAMSON, M. L. Evolutionary biology of the Oxyurida (Nematoda): biofacies of a haplodiploid taxon. Advances in Parasitology, v. 28, p. $175-228,1989$.

AMATO, J. F. R. et al. Trypanoxyuris (Trypanoxyuris) minutus associated with the death of a wild southern brown howler monkey, Alouatta guariba clamitans, in Rio Grande do Sul, Brazil. Arquivos do Instituto Biológico, v. 69, n. 4, p. 99-102, 2002.

ANDERSON, R. C. Nematode parasites of vertebrates: their development and transmission. 2. ed. Oxford: CABI Publishing, 2000. 672 p.

BUSH, A. O. et al. Parasitology meets ecology on its own terms: Margolis et al. revisited. Journal of Parasitology, v. 83, n. 4, p. 575-583, 1997.

GREGORIN, R. Taxonomia e variação geográfica das espécies do gênero Alouatta Lacépède (Primates, Atelidae) no Brasil. Revista Brasileira de Zoologia, v. 23, n. 1, p. 64-144, 2006.

HUGOT, J. P.; GARDNER, S. L.; MORAND, S. The enterobiinae subfam: nov. (Nematoda, Oxyurida) pinworm parasites of primates and rodents. International Journal for Parasitology, v. 26, n. 2, p. 147-159, 1996.

HUGOT, J. P.; VAUCHER, C. Sur lê genre Trypanoxyuris (Oxyuridae, Nematoda): sous-genre Trypanoxyuris parasite de primates cebidae et atelidae (suíte) : étude morphologique de Trypanoxyuris callicebi $\mathrm{n}$. sp. Bulletin du Museum National d'Histoire Naturelle Section A, v. 7, n. 3, p. 633-636, 1985.

STONER, K. E. Prevalence and intensity of intestinal parasites in mantled howling monkeys (Alouatta palliata) in northeastern Costa Rica: implications for conservation biology. Conservation Biology, v. 10, n. 2 , p. $539-546,1996$.

STUART, M. et al. Parasites of wild howlers (Alouatta spp.). International Journal of Primatology, v. 19, n. 3, p. 493-512, 1998.

TRAVASSOS, L. Revisão do gênero Enterobius Leach, 1852. Fauna brasiliense, Museu Nacional do Rio de Janeiro, v. 2, p. 11, 1925.

TRAVASSOS, L.; PINTO, C.; MUNIZ, J. Excursão cientifica ao estado de Mato Grosso na zona do Pantanal (margens dos rios S. Lourenço e Cuiabá) realizada em 1922. Memórias do Instituto Oswaldo Cruz, v. 20, n. 2, p. 249-269, 1927.

VICENTE, J. J. et al. Nematóides do Brasil: nematóides de mamíferos. Revista Brasileira de Zoologia, v. 14, supl. 1, p. 1-452, 1997.

YAMAGUTI, S. Systema helminthum 3: the nematodes of vertebrates. New York: Interscience Publishers, 1961. 1261 p. 